IFUM-852-FT

\title{
Renormalization of the Non-Linear Sigma Model in Four Dimensions. A two-loop example.
}

\author{
Ruggero Ferrari ${ }^{1}$ and Andrea Quadri ${ }^{2}$ \\ Phys. Dept. University of Milan, via Celoria 16, 20133 Milan, Italy \\ and I.N.F.N., sezione di Milano
}

\begin{abstract}
The renormalization procedure of the non-linear $S U(2)$ sigma model in $D=4$ proposed in Refs. [1, 2] is here tested in a truly non-trivial case where the non-linearity of the functional equation is crucial. The simplest example, where the non-linear term contributes, is given by the two-loop amplitude involving the insertion of two $\phi_{0}$ (the constraint of the non-linear sigma model) and two flat connections. In this case we verify the validity of the renormalization procedure: the recursive subtraction of the pole parts at $D=4$ yields amplitudes that satisfy the defining functional equation. As a by-product we give a formal proof that in $D$ dimensions (without counterterms) the Feynman rules provide a perturbative symmetric solution.
\end{abstract}

\footnotetext{
${ }^{1}$ E-mail address: ruggero.ferrari@mi.infn.it

${ }^{2}$ E-mail address: andrea.quadri@mi.infn.it
} 


\section{Introduction}

In Refs. [1, 2] it was proposed to quantize the non-linear sigma model in $D=4$ by embedding the pion fields in a flat connection in order to solve the long-standing problem [3]-[9] of a symmetric removal of the divergences. The theory is defined by the functional equation for the connected amplitudes

$$
\begin{aligned}
\mathcal{S}(W) & =\left(\frac{m_{D}^{2}}{2} \partial^{\mu} \frac{\delta W}{\delta J_{a}^{\mu}}+g^{2} \frac{\delta W}{\delta K_{a}} K_{0}-K_{a} \frac{\delta W}{\delta K_{0}}-g \epsilon_{a b c} K_{b} \frac{\delta W}{\delta K_{c}}\right. \\
& \left.+2 \mathcal{D}\left[\frac{\delta W}{\delta J}\right]_{a b}^{\mu} J_{b \mu}\right)(x)=0
\end{aligned}
$$

or for 1-PI amplitudes

$$
\begin{aligned}
(\Gamma, \Gamma)= & \left(\frac{m_{D}^{2}}{2} \partial^{\mu} \frac{\delta \Gamma}{\delta J_{a}^{\mu}}+g^{2} \phi_{a} K_{0}+\frac{\delta \Gamma}{\delta K_{0}} \frac{\delta \Gamma}{\delta \phi_{a}}+g \epsilon_{a b c} \frac{\delta \Gamma}{\delta \phi_{b}} \phi_{c}\right. \\
& \left.+2 \mathcal{D}\left[\frac{\delta \Gamma}{\delta J}\right]_{a b}^{\mu} J_{b \mu}\right)(x)=0
\end{aligned}
$$

with

$$
\mathcal{D}[X]_{a b}^{\mu}=\partial^{\mu} \delta_{a b}-g \epsilon_{a b c} X_{c}^{\mu} .
$$

Moreover it was conjectured and shown [1] in a few examples that standard perturbation theory in $D$-dimensions gives amplitudes that satisfy the equation without any subtractions. The limit to $D=4$ is divergent and needs subtraction of the poles of the Laurent expansion. This is done by using the properly normalized 1-PI amplitudes involving only insertions of flat connections and the constrained field $\phi_{0}$, i.e. those amplitudes which are on the top of the hierarchy implicit in eq.(2).

This procedure is consistent if the subtraction procedure (use of counterterms in the Feynman rules) does not violate eq.(2) which is a non-linear equation in the bilinear term

$$
\frac{\delta \Gamma}{\delta K_{0}(x)} \frac{\delta \Gamma}{\delta \phi_{a}(x)}
$$

At one-loop level this problem has been addressed in the paper [2]. There it was shown that at one-loop level eq.(2) becomes linear and the counterterms given by the subtraction procedure are a solution of the equation. So at the one-loop level there is no anomaly. Moreover in the same paper the most general local finite renormalization compatible with the functional equation 
has been classified (the number of these free parameters is finite, a property that we indicated as weak power-counting theorem).

The simplest two-loop example where the bilinear term in eq.(4) is nonzero is the four-point-amplitude involving two flat connections and two constrained fields. In this case eq.(2) becomes

$$
\begin{aligned}
& \frac{m_{D}^{2}}{2} \partial^{\mu} \Gamma_{J_{a}^{\mu} J K_{0} K_{0}}^{(2)}+m_{D} \Gamma_{\phi_{a} J K_{0} K_{0}}^{(2)}+\Gamma_{K_{0} K_{0} K_{0}}^{(2)} \Gamma_{\phi_{a} J}^{(0)} \\
& +\Gamma_{K_{0} K_{0}}^{(1)} \Gamma_{\phi_{a} K_{0} J}^{(1)}=0 .
\end{aligned}
$$

In this paper we show that the subtraction procedure for the $D=4$ limit provides amplitudes that satisfy eq.(15). We do not evaluate explicitly the second-order counterterms, rather we study eq.(15) where only the first order counterterms are introduced and we prove that the local second order subtraction restores the validity of the equation.

The paper is organized as follows. In Sect. 2 we consider the consequences of the introduction of the first order counterterms on the functional equation at the two-loop level. In Sect. 3 we prove that the contributuion of the graphs with no counterterms cancel exactly. This result is obtained by a quantum action principle for the unsubtracted amplitudes. In Sect. 4 we consider the contribution of all graphs containing one-loop counterterms. The result of these two sections allows the evaluation of the breaking term of the functional equation which turns out to be local. This is described in Sect. 5. We conclude that the breaking term is removed completely by the subtraction procedure and therefore that the functional equation is stable under renormalization in this particular example.

\section{Breaking of the functional equation}

The object of our investigation is the amplitude $\Gamma_{J J K_{0} K_{0}}^{(2)}$ at the two-loop level in the limit $D=4$. The contribution to this amplitude without insertion of counterterms will be denoted by $\Gamma_{J J K_{0} K_{0}}^{(2,0)}$ while the amplitude with one counterterms insertion will be denoted by $\Gamma_{J J K_{0} K_{0}}^{(2,1)}$. The two contributions yield an amplitude $\Gamma_{J J K_{0} K_{0}}^{(2,0)}+\Gamma_{J J K_{0} K_{0}}^{(2,1)}$ which is expected to develop a pole in the limit $D=4$, thus necessitating a further and last subtraction. However before doing this last step we consider the breaking of the 
functional equation caused by the one-loop counterterm insertion

$$
\begin{aligned}
& \frac{m_{D}^{2}}{2} \partial^{\mu}\left(\Gamma_{J_{a}^{\mu}}^{(2,0)}\left[J J K_{0} K_{0}\right]+\Gamma_{J_{a}^{\mu}}^{(2,1)}\left[J J K_{0} K_{0}\right]\right) \\
& +m_{D}\left(\Gamma_{\phi_{a}}^{(2,0)}\left[\phi J K_{0} K_{0}\right]+\Gamma_{\phi_{a}}^{(2,1)}\left[\phi J K_{0} K_{0}\right]\right) \\
& +\left(\Gamma_{K_{0}}^{(2,0)}\left[K_{0} K_{0} K_{0}\right]+\Gamma_{K_{0}}^{(2,1)}\left[K_{0} K_{0} K_{0}\right]\right) \Gamma_{\phi_{a}}^{(0)}[\phi J] \\
& +\left(\Gamma_{K_{0}}^{(1,0)}\left[K_{0} K_{0}\right]+\hat{\Gamma}_{K_{0}}^{(1)}\left[K_{0} K_{0}\right]\right)\left(\Gamma_{\phi_{a}}^{(1,0)}\left[\phi K_{0} J\right]+\hat{\Gamma}_{\phi_{a}}^{(1)}\left[\phi K_{0} J\right]\right) \\
& \quad=\Delta^{(2)}\left[J J K_{0}\right] .
\end{aligned}
$$

The crucial point is the evaluation of $\Delta^{(2)}$. It is important to show that it is local and that it is removed by the subtraction procedure. It is worth to recall here the subtraction procedure at $D=4$. This is performed recursively, i.e. after the $(n-1)$-subtraction has been performed the resulting amplitudes are properly normalized and expanded in a Laurent series at $D=4$. Finally the pole parts, which are local, are removed by introducing the counterterms in the effective action.

It is very crucial to respect the hierarchy and to normalize the amplitudes. The functional equation shows that one has to make finite only the amplitudes involving derivatives w.r.t $J_{\mu}$ and $K_{0}$. In fact all the other amplitudes are derived by subsequent functional differentiation w.r.t the field $\phi_{a}$. For dimensional reasons the amplitudes are normalized by

$$
\left(\frac{m_{D}}{m}\right)^{2(n-1)} \Gamma_{J_{a_{1}}^{\mu_{1} \ldots J_{n}}}^{\mu_{n}}=m^{(n-1)(D-4)} \Gamma_{J_{a_{1}}^{\mu_{1}} \ldots J_{a_{n}}^{\mu_{n}}} .
$$

Once again we stress that the very definition of the theory at $D=4$ crucially depends on the subtraction procedure outlined above and our goal is to prove that it does not spoil the functional equation (no anomalies).

\section{Amplitudes without counterterms}

It has been conjectured in [1] that the unsubtracted amplitudes satisfy the functional equation. It is worth then to consider a subset of the terms appearing in eq.(6), i.e. those involving amplitudes $\Gamma^{(1,0)}$ and $\Gamma^{(2,0)}$. In this section we in fact demonstrate the correctness of the conjecture by using quantum action principle arguments [10]-12].

We use the generating functional

$$
Z\left[K_{a}, K_{0}, J_{\mu}\right]=\exp \left(\left.i \Gamma_{\text {int }}^{(0)}\left[\phi, K_{0}, J\right]\right|_{\phi_{a}=\frac{1}{i} \frac{\delta}{\delta K_{a}}}\right) \exp \left(\frac{i}{2} \int K_{a} \Delta_{F} K_{a}\right)
$$


where tadpole contributions are discharged. Then we apply the operator $\mathcal{S}$ in eq.(11) to the connected generating functional

$$
W=-i \ln Z
$$

and we get

$$
\begin{aligned}
\mathcal{S}(W)=i( & \frac{m_{D}^{2}}{2} \partial^{\mu} \frac{\delta \Gamma^{(0)}}{\delta J_{\mu}^{a}}+g^{2} \phi_{a} K_{0} \\
& +\frac{\delta \Gamma^{(0)}}{\delta K_{0}} \frac{\delta \Gamma^{(0)}}{\delta \phi_{a}}+g \epsilon_{a b c} \frac{\delta \Gamma^{(0)}}{\delta \phi_{b}} \phi_{c} \\
& \left.+2 \mathcal{D}\left[\frac{\delta \Gamma^{(0)}}{\delta J}\right]_{a b}^{\mu} J_{b \mu}\right) \cdot W\left[K_{a}, K_{0}, J_{\mu}\right]
\end{aligned}
$$

where the $\cdot$ denotes the insertion. Since

$$
\begin{aligned}
\left(\Gamma^{(0)}, \Gamma^{(0)}\right)= & \left(\frac{m_{D}^{2}}{2} \partial^{\mu} \frac{\delta \Gamma^{(0)}}{\delta J_{a}^{\mu}}+g^{2} \phi_{a} K_{0}+\frac{\delta \Gamma^{(0)}}{\delta K_{0}} \frac{\delta \Gamma^{(0)}}{\delta \phi_{a}}+g \epsilon_{a b c} \frac{\delta \Gamma^{(0)}}{\delta \phi_{b}} \phi_{c}\right. \\
& \left.+2 \mathcal{D}\left[\frac{\delta \Gamma^{(0)}}{\delta J}\right]_{a b}^{\mu} J_{b \mu}\right)(x)=0
\end{aligned}
$$

we obtain

$$
\mathcal{S}(W)=0
$$

All the insertions in the R.H.S. of eq. (10) mediated by one-particle states cancel out. Therefore all the insertions of the composite operators in the R.H.S. of eq.(10) can be replaced by insertions on $\Gamma$. Hence we get

$$
\begin{aligned}
& i\left(\frac{m_{D}^{2}}{2} \partial^{\mu} \frac{\delta \Gamma^{(0)}}{\delta J_{\mu}^{a}}+g^{2} \phi_{a} K_{0}+\frac{\delta \Gamma^{(0)}}{\delta K_{0}} \frac{\delta \Gamma^{(0)}}{\delta \phi_{a}}+g \epsilon_{a b c} \frac{\delta \Gamma^{(0)}}{\delta \phi_{b}} \phi_{c}\right. \\
& \left.+2 \mathcal{D}\left[\frac{\delta \Gamma^{(0)}}{\delta J}\right]_{a b}^{\mu} J_{b \mu}\right) \cdot \Gamma\left[\phi_{a}, K_{0}, J_{\mu}\right]=0
\end{aligned}
$$

This equation can be used to perform a detailed diagrammatic analysis (see Appendix (A) of the validity of the functional equation (2) at two-loop level for the unsubtracted amplitudes:

$$
\begin{aligned}
& \frac{m_{D}^{2}}{2} \partial_{\mu} \frac{\delta \Gamma^{(2,0)}\left[J J K_{0} K_{0}\right]}{\delta J_{a}^{\mu}}+m_{D} \frac{\delta \Gamma^{(2,0)}\left[\phi J K_{0} K_{0}\right]}{\delta \phi_{a}} \\
& \quad+\frac{\delta \Gamma^{(2,0)}\left[K_{0} K_{0} K_{0}\right]}{\delta K_{0}} \frac{\delta \Gamma^{(0)}[\phi J]}{\delta \phi_{a}}+\frac{\delta \Gamma^{(1,0)}\left[K_{0} K_{0}\right]}{\delta K_{0}} \frac{\delta \Gamma^{(1,0)}\left[\phi_{a} J K_{0}\right]}{\delta \phi_{a}}=0 .
\end{aligned}
$$




\section{Amplitudes with one-loop counterterms}

The surprising result of this section is that the sum of all the amplitudes carrying a one-loop counterterm and that can potentially give a non-local contribution to $\Delta^{(2)}$ in eq.(6) do in fact sum up to zero:

$$
\begin{aligned}
& \frac{m_{D}^{2}}{2} \partial^{\mu} \Gamma_{J_{a}^{\mu}}^{(2,1)}\left[J J K_{0} K_{0}\right]+m_{D} \Gamma_{\phi_{a}}^{(2,1)}\left[K_{0} K_{0} J \phi\right] \\
& +\Gamma_{K_{0}}^{(2,1)}\left[K_{0} K_{0} K_{0}\right] \Gamma_{\phi_{a}}^{(0)}[\phi J]+\Gamma_{K_{0}}^{(1,0)}\left[K_{0} K_{0}\right] \hat{\Gamma}_{\phi_{a}}^{(1)}\left[\phi K_{0} J\right] \\
& +\hat{\Gamma}_{K_{0}}^{(1)}\left[K_{0} K_{0}\right] \Gamma_{\phi_{a}}^{(1,0)}\left[J K_{0} \phi\right]=0 .
\end{aligned}
$$

We first give a general argument based on the quantum action principle for the generating functional of the amplitudes which now includes the counterterms at one-loop among the Feynman rules. Then eq.(8) now becomes

$Z_{1 R}\left[K_{a}, K_{0}, J\right]=\exp \left(\left.\left(i \Gamma_{i n t}^{(0)}+i \hat{\Gamma}^{(1)}\right)\right|_{\phi_{a}=\frac{1}{i} \frac{\delta}{\delta K_{a}}}\right) \exp \left(\frac{i}{2} \int K_{a} \Delta_{F} K_{a}\right)$.

The functional equation for the connected amplitudes $W_{1 R}=-i \ln Z_{1 R}$ now becomes

$$
\begin{aligned}
\mathcal{S}\left(W_{1 R}\right) & =\left(\frac{m_{D}^{2}}{2} \partial^{\mu} \frac{\delta\left[\Gamma^{(0)}+\hat{\Gamma}^{(1)}\right]}{\delta J_{a}^{\mu}(x)}+g^{2} \phi_{a}(x) K_{0}(x)\right. \\
& \left.+\frac{\delta\left[\Gamma^{(0)}+\hat{\Gamma}^{(1)}\right]}{\delta K_{0}(x)} \frac{\delta\left[\Gamma^{(0)}+\hat{\Gamma}^{(1)}\right]}{\delta \phi_{a}(x)}+2 \mathcal{D}\left[\frac{\delta\left[\Gamma^{(0)}+\hat{\Gamma}^{(1)}\right]}{\delta J}\right]_{\mu}^{a b} J_{b}^{\mu}(x)\right) \cdot W_{1 R} .
\end{aligned}
$$

The counterterms obey the linearized form of eq.(21) (see Refs. [1, 2]):

$\frac{m_{D}^{2}}{2} \partial^{\mu} \frac{\delta \hat{\Gamma}^{(1)}}{\delta J_{a}^{\mu}(x)}+\frac{\delta \Gamma^{(0)}}{\delta K_{0}(x)} \frac{\delta \hat{\Gamma}^{(1)}}{\delta \phi_{a}(x)}+\frac{\delta \hat{\Gamma}^{(1)}}{\delta K_{0}(x)} \frac{\delta \Gamma^{(0)}}{\delta \phi_{a}(x)}+2 \mathcal{D}\left[\frac{\delta \hat{\Gamma}^{(1)}}{\delta J}\right]_{\mu}^{a b} J_{b}^{\mu}(x)=0$.

Therefore as a consequence of the quantum action principle in eq.(17) and of eqs.(11) and (18) we get

$$
\mathcal{S}\left(W_{1 R}\right)=\frac{\delta \hat{\Gamma}^{(1)}}{\delta K_{0}(x)} \frac{\delta \hat{\Gamma}^{(1)}}{\delta \phi_{a}(x)} \cdot W_{1 R}
$$

This result can be explicitly verified in our particular example. In appendix $\mathrm{B}$ we prove diagrammatically the validity of eq.(15) and consequently that of eq. (19) by the evaluation of $\Delta^{(2)}$ in eq.(6). Two different checks are 
needed. First one verifies that indeed the insertion of the L.H.S. in eq. (18) is zero:

$$
\begin{gathered}
\left(\frac{m_{D}^{2}}{2} \partial^{\mu} \frac{\delta \hat{\Gamma}^{(1)}}{\delta J_{a}^{\mu}(x)}+\frac{\delta \Gamma^{(0)}}{\delta K_{0}(x)} \frac{\delta \hat{\Gamma}^{(1)}}{\delta \phi_{a}(x)}+\frac{\delta \hat{\Gamma}^{(1)}}{\delta K_{0}(x)} \frac{\delta \Gamma^{(0)}}{\delta \phi_{a}(x)}+2 \mathcal{D}\left[\frac{\delta \hat{\Gamma}^{(1)}}{\delta J}\right]_{\mu}^{a b} J_{b}^{\mu}(x)\right) \\
\cdot W_{1 R}=0 .
\end{gathered}
$$

Again all the insertions in the L.H.S. of eq. (20) mediated by one-particle states cancel out. Therefore all the insertions of the composite operators in the R.H.S. of eq.(20) can be replaced by insertions on $\Gamma$. Hence we get

$$
\begin{aligned}
&\left(\frac{m_{D}^{2}}{2} \partial^{\mu}\right.\left.\frac{\delta \hat{\Gamma}^{(1)}}{\delta J_{a}^{\mu}(x)}+\frac{\delta \Gamma^{(0)}}{\delta K_{0}(x)} \frac{\delta \hat{\Gamma}^{(1)}}{\delta \phi_{a}(x)}+\frac{\delta \hat{\Gamma}^{(1)}}{\delta K_{0}(x)} \frac{\delta \Gamma^{(0)}}{\delta \phi_{a}(x)}+2 \mathcal{D}\left[\frac{\delta \hat{\Gamma}^{(1)}}{\delta J}\right]_{\mu}^{a b} J_{b}^{\mu}(x)\right) \\
& \cdot \Gamma_{1 R}=0 .
\end{aligned}
$$

Moreover one needs to check that the insertion of the L.H.S. of eq.(11) is zero also at one-loop level, i.e.

$$
\left(\Gamma^{(0)}, \Gamma^{(0)}\right) \cdot W_{1 R}=0 .
$$

In the above equation we can again restrict ourselves to the 1-PI amplitudes since the insertions mediated by one-particles states in the L.H.S. cancel out, so that

$$
\left(\Gamma^{(0)}, \Gamma^{(0)}\right) \cdot \Gamma_{1 R}=0 .
$$

The diagrammatic evaluation of the L.H.S. eq.(15) is finally achieved by a combined use of eq.(21) and eq.(23).

\section{Removal of the breaking $\Delta^{(2)}\left[J K_{0} K_{0}\right]$}

The result of the previous sections has shown that the breaking of the functional equation at the two-loop level after the introduction of the one-loop level counterterms is given by

$$
\Delta^{(2)}=\frac{\delta \hat{\Gamma}^{(1)}}{\delta K_{0}(x)} \frac{\delta \hat{\Gamma}^{(1)}}{\delta \phi_{a}(x)} \cdot \Gamma_{1 R} .
$$

In order to proceed further we specialize to the case we are dealing with

$$
\begin{aligned}
& \Delta^{(2)}\left[J K_{0} K_{0}\right]=\hat{\Gamma}_{K_{0}(x)}^{(1)}\left[K_{0} K_{0}\right] \hat{\Gamma}_{\phi_{a}(x)}^{(1)}\left[\phi J K_{0}\right] \cdot 1 \\
& \quad=\left(\frac{1}{D-4}\right)^{2} \frac{6 g^{8}}{(4 \pi)^{4}} \frac{1}{m^{4} m_{D}^{2}} K_{0}(x)\left(-4 \partial^{\mu} K_{0}(x) J_{a \mu}(x)-K_{0}(x) \partial J_{a}(x)\right) .
\end{aligned}
$$


This is of course a local insertion and then the last very crucial point is to verify that after the subtraction procedure it disappears (not even finite parts are left over). In order to prove that this is indeed the case one must remember that the subtraction of the poles has to be performed on the normalized amplitudes as stated in eq.(17). Thus we have to expand in a Laurent series both sides of the equation

$$
\begin{aligned}
& \frac{1}{m^{2}}\left(\frac{m_{D}}{m}\right)^{2}\left(\frac{m_{D}^{2}}{2} \partial^{\mu} \frac{\delta \Gamma^{(2)}\left[J J K_{0} K_{0}\right]}{\delta J_{a}^{\mu}(x)}+m_{D} \frac{\delta \Gamma^{(2)}\left[\phi J K_{0} K_{0}\right]}{\delta \phi_{a}(x)}\right. \\
& \left.\quad+\frac{\delta \Gamma^{(1)}\left[K_{0} K_{0}\right]}{\delta K_{0}(x)} \frac{\delta \Gamma^{(1)}\left[\phi K_{0} J\right]}{\delta \phi_{a}(x)}+\frac{\delta \Gamma^{(2)}\left[K_{0} K_{0} K_{0}\right]}{\delta K_{0}(x)} \frac{\delta \Gamma^{(0)}[\phi J]}{\delta \phi_{a}(x)}\right) \\
& \quad=\frac{1}{m^{2}}\left(\frac{m_{D}}{m}\right)^{2} \Delta^{(2)}\left[K_{0} K_{0} J\right] .
\end{aligned}
$$

In the R.H.S. $m_{D}^{2}$ cancels out and therefore the R.H.S. contains a pure double pole with no finite parts left over. The pole part disappears after the subtraction procedure has been performed on the L.H.S. of the above equation. Once again we stress that the subtraction procedure has to be applied in the L.H.S. of eq.(26) to each of the terms $\left(\frac{m_{D}}{m}\right)^{4} \Gamma^{(2)}\left[J J K_{0} K_{0}\right]$, $\left(\frac{m_{D}}{m}\right)^{3} \Gamma^{(2)}\left[\phi J K_{0} K_{0}\right]$ and $\left(\frac{m_{D}}{m}\right) \Gamma^{(2)}\left[K_{0} K_{0} K_{0}\right]$ (notice that $\frac{\delta \Gamma^{(0)}[\phi J]}{\delta \phi_{a}(x)}=\frac{2}{m_{D}} J_{a}^{\mu} \partial_{\mu} \phi_{a}$ ).

\section{Conclusions and outlook}

The aim of this paper is to show on a specific example that the subtraction procedure at $D=4$ is symmetric, i.e. the functional equation is stable under renormalization at the two-loop level. The proof is based on the evaluation of the breaking term at two loops after the introduction of firstorder counterterms. We have been able to show that the breaking is a local insertion. Moreover it is subtracted completely when we remove the overall poles in $D=4$ from the two-loop amplitudes corrected by the insertion of one-loop counterterms.

The technique used can be probably applied to a general case since we use a recursive method. As a by-product we have also shown that the perturbative series in generic dimension $D$ satisfies the functional equation. After these results the theory looks promising and one can think to some phenomenological applications and to a more general approach (systematic use of cohomological methods to classify finite renormalizations) to the renormalization of the non-linear sigma model. 
Many open questions can be addressed at this point. We consider of particular interest the possibility to interpret finite renormalizations as a kind of deformation of the geometry of the $\phi$-manifold [13]-15] also in $D=4$.

\section{A Diagrammatic analysis of the functional equa- tion for unsubtracted amplitudes}

We evaluate the insertion in the L.H.S. of eq.(13) in the relevant 1-PI sector spanned by two $K_{0}$ and one $J_{\mu}$ (see eq.(14)).

The functional equation (111) yields

$$
\begin{gathered}
\frac{m_{D}^{2}}{2} \partial^{\mu} \frac{\delta \Gamma^{(0)}[J \phi \phi \phi]}{\delta J_{a}^{\mu}(x)}+m_{D} \frac{\delta \Gamma^{(0)}[\phi \phi \phi \phi]}{\delta \phi_{a}(x)}+\frac{\delta \Gamma^{(0)}\left[K_{0} \phi \phi\right]}{\delta K_{0}(x)} \frac{\delta \Gamma^{(0)}[\phi \phi]}{\delta \phi_{a}(x)}=0, \\
\frac{m_{D}^{2}}{2} \partial^{\mu} \frac{\delta \Gamma^{(0)}[J \phi \phi]}{\delta J_{a}^{\mu}(x)}+g \epsilon_{a b c} \frac{\delta \Gamma^{(0)}[\phi \phi]}{\delta \phi_{b}(x)} \phi_{c}(x)=0 \\
m_{D} \frac{\delta \Gamma^{(0)}\left[K_{0} \phi \phi \phi \phi\right]}{\delta \phi_{a}(x)}+\frac{\delta \Gamma^{(0)}\left[K_{0} \phi \phi\right]}{\delta K_{0}(x)} \frac{\delta \Gamma^{(0)}\left[K_{0} \phi \phi\right]}{\delta \phi_{a}(x)}=0 \\
m_{D} \frac{\delta \Gamma^{(0)}[J \phi \phi \phi \phi \phi]}{\delta \phi_{a}(x)}+\frac{\delta \Gamma^{(0)}\left[K_{0} \phi \phi\right]}{\delta K_{0}(x)} \frac{\delta \Gamma^{(0)}[J \phi \phi \phi]}{\delta \phi_{a}(x)} \\
+\frac{\delta \Gamma^{(0)}\left[K_{0} \phi \phi \phi \phi\right]}{\delta K_{0}(x)} \frac{\delta \Gamma^{(0)}[J \phi]}{\delta \phi_{a}(x)}=0, \\
m_{D} \frac{\delta \Gamma^{(0)}[J \phi \phi \phi]}{\delta \phi_{a}(x)}+\frac{\delta \Gamma^{(0)}\left[K_{0} \phi \phi\right]}{\delta K_{0}(x)} \frac{\delta \Gamma^{(0)}[J \phi]}{\delta \phi_{a}(x)} \\
+g \epsilon_{a b c} \frac{\delta \Gamma^{(0)}[J \phi \phi]}{\delta \phi_{b}(x)} \phi_{c}(x)-2 g \epsilon_{a b c} \frac{\delta \Gamma^{(0)}[J \phi \phi]}{\delta J_{c}^{\mu}(x)} J_{b}^{\mu}(x)=0 .
\end{gathered}
$$

From eq. 27) we obtain (in $\langle\cdot\rangle$ we omit the $T$-symbol in order to simplify the notations)

$$
\begin{gathered}
\frac{1}{2}\left\langle\left(\frac{m_{D}^{2}}{2} \partial^{\mu} \frac{\delta \Gamma^{(0)}[J \phi \phi \phi]}{\delta J_{a}^{\mu}(x)}+m_{D} \frac{\delta \Gamma^{(0)}[\phi \phi \phi \phi]}{\delta \phi_{a}(x)}+\frac{\delta \Gamma^{(0)}\left[K_{0} \phi \phi\right]}{\delta K_{0}(x)} \frac{\delta \Gamma^{(0)}[\phi \phi]}{\delta \phi_{a}(x)}\right)\right. \\
\left.\Gamma^{(0)}[J \phi \phi \phi] \Gamma^{(0)}\left[K_{0} \phi \phi\right] \Gamma^{(0)}\left[K_{0} \phi \phi\right]\right\rangle \\
-\left\langle\left\langle\left(\frac{m_{D}^{2}}{2} \partial^{\mu} \frac{\delta \Gamma^{(0)}[J \phi \phi \phi]}{\delta J_{a}^{\mu}(x)}+m_{D} \frac{\delta \Gamma^{(0)}[\phi \phi \phi \phi]}{\delta \phi_{a}(x)}+\frac{\delta \Gamma^{(0)}\left[K_{0} \phi \phi\right]}{\delta K_{0}(x)} \frac{\delta \Gamma^{(0)}[\phi \phi]}{\delta \phi_{a}(x)}\right) \Gamma^{(0)}\left[K_{0} \phi \phi\right]\right\rangle\right. \\
\left.\left\langle\Gamma^{(0)}[J \phi \phi \phi] \Gamma^{(0)}\left[K_{0} \phi \phi\right]\right\rangle\right\rangle=0 .
\end{gathered}
$$




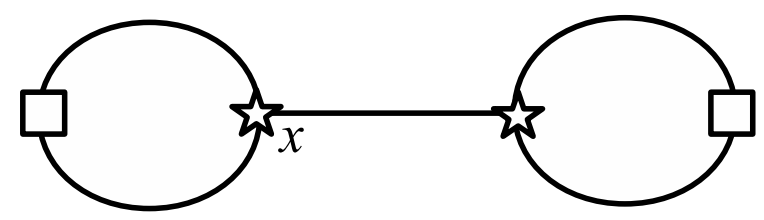

Figure 1: Spurious (non 1-PI) contribution in the insertion of eq. (32) (stars and boxes are insertions of flat connections and of $\phi_{0}$ respectively).

The second term in the above equation subtracts the connected but not 1-PI contribution, depicted in Fig. 1, which enters in the set of contractions in the first term of eq. (32).

Furthermore we obtain from eq.(28)

$$
\begin{aligned}
\left\langle\left(\frac{m_{D}^{2}}{2} \partial^{\mu} \frac{\delta \Gamma^{(0)}[J \phi \phi]}{\delta J_{a}^{\mu}(x)}+g \epsilon_{a b c} \frac{\delta \Gamma^{(0)}[\phi \phi]}{\delta \phi_{b}(x)} \phi_{c}(x)\right)\right. \\
\left.\Gamma^{(0)}[J \phi \phi] \Gamma^{(0)}\left[K_{0} \phi \phi \phi \phi\right] \Gamma^{(0)}\left[K_{0} \phi \phi\right]\right\rangle=0
\end{aligned}
$$

and also

$$
\begin{aligned}
\frac{i}{2}\left\langle\left(\frac{m_{D}^{2}}{2} \partial^{\mu} \frac{\delta \Gamma^{(0)}[J \phi \phi]}{\delta J_{a}^{\mu}(x)}+g \epsilon_{a b c} \frac{\delta \Gamma^{(0)}[\phi \phi]}{\delta \phi_{b}(x)} \phi_{c}(x)\right)\right. \\
\left.\Gamma^{(0)}[J \phi \phi] \Gamma^{(0)}\left[K_{0} \phi \phi\right] \Gamma^{(0)}\left[K_{0} \phi \phi\right] \Gamma^{(0)}[\phi \phi \phi \phi]\right\rangle=0 .
\end{aligned}
$$

From eq.(29) we get

$$
-i\left\langle\left(m_{D} \frac{\delta \Gamma^{(0)}\left[K_{0} \phi \phi \phi \phi\right]}{\delta \phi_{a}(x)}+\frac{\delta \Gamma^{(0)}\left[K_{0} \phi \phi\right]}{\delta K_{0}(x)} \frac{\delta \Gamma^{(0)}\left[K_{0} \phi \phi\right]}{\delta \phi_{a}(x)}\right) \Gamma^{(0)}\left[K_{0} \phi \phi\right] \Gamma^{(0)}[J \phi \phi \phi]\right\rangle=0 .
$$

From eq.(30) we get

$$
\begin{gathered}
-\frac{i}{2}\left\langle\left(m_{D} \frac{\delta \Gamma^{(0)}[J \phi \phi \phi \phi \phi]}{\delta \phi_{a}(x)}+\frac{\delta \Gamma^{(0)}\left[K_{0} \phi \phi\right]}{\delta K_{0}(x)} \frac{\delta \Gamma^{(0)}[J \phi \phi \phi]}{\delta \phi_{a}(x)}+\frac{\delta \Gamma^{(0)}\left[K_{0} \phi \phi \phi \phi\right]}{\delta K_{0}(x)} \frac{\delta \Gamma^{(0)}[J \phi]}{\delta \phi_{a}(x)}\right)\right. \\
\left.\Gamma^{(0)}\left[K_{0} \phi \phi\right] \Gamma^{(0)}\left[K_{0} \phi \phi\right]\right\rangle=0 .
\end{gathered}
$$


In order to decompose further the diagrams contributing to the above insertions we need the fact that

$$
\square_{x} \Delta_{F}(x-y)=-i \delta^{D}(x-y)
$$

By using eq.(37) the third term in the first line of eq.(32) becomes

$$
\begin{aligned}
\frac{1}{2}\langle & \left.\frac{\delta \Gamma^{(0)}\left[K_{0} \phi \phi\right]}{\delta K_{0}(x)} \frac{\delta \Gamma^{(0)}[\phi \phi]}{\delta \phi_{a}(x)} \Gamma^{(0)}[J \phi \phi \phi] \Gamma^{(0)}\left[K_{0} \phi \phi\right] \Gamma^{(0)}\left[K_{0} \phi \phi\right]\right\rangle \\
= & -\frac{1}{2}\left\langle\frac{\delta \Gamma^{(0)}\left[K_{0} \phi \phi\right]}{\delta K_{0}(x)} \square \phi_{a}(x) \Gamma^{(0)}[J \phi \phi \phi] \Gamma^{(0)}\left[K_{0} \phi \phi\right] \Gamma^{(0)}\left[K_{0} \phi \phi\right]\right\rangle \\
= & i\left\langle\frac{\delta \Gamma^{(0)}\left[K_{0} \phi \phi\right]}{\delta \phi_{a}(x)} \Gamma^{(0)}\left[K_{0} \phi \phi\right] \frac{\delta \Gamma^{(0)}\left[K_{0} \phi \phi\right]}{\delta K_{0}(x)} \Gamma^{(0)}[J \phi \phi \phi]\right\rangle \\
& +\frac{1}{2} i\left\langle\Gamma^{(0)}\left[K_{0} \phi \phi\right] \Gamma^{(0)}\left[K_{0} \phi \phi\right] \frac{\delta \Gamma^{(0)}\left[K_{0} \phi \phi\right]}{\delta K_{0}(x)} \frac{\delta \Gamma^{(0)}[J \phi \phi \phi]}{\delta \phi_{a}(x)}\right\rangle .
\end{aligned}
$$

In a similar fashion the third term in the third line of the L.H.S. of eq.(32) can be simplified as follows:

$$
\begin{gathered}
-\left\langle\left\langle\frac{\delta \Gamma^{(0)}\left[K_{0} \phi \phi\right]}{\delta K_{0}(x)} \frac{\delta \Gamma^{(0)}[\phi \phi]}{\delta \phi_{a}(x)} \Gamma^{(0)}\left[K_{0} \phi \phi\right]\right\rangle\left\langle\Gamma^{(0)}[J \phi \phi \phi] \Gamma^{(0)}\left[K_{0} \phi \phi\right]\right\rangle\right\rangle \\
=\left\langle\left\langle\frac{\delta \Gamma^{(0)}\left[K_{0} \phi \phi\right]}{\delta K_{0}(x)} \square \phi_{a}(x) \Gamma^{(0)}\left[K_{0} \phi \phi\right]\right\rangle\left\langle\Gamma^{(0)}[J \phi \phi \phi] \Gamma^{(0)}\left[K_{0} \phi \phi\right]\right\rangle\right\rangle \\
=-i\left\langle\left\langle\frac{\delta \Gamma^{(0)}\left[K_{0} \phi \phi\right]}{\delta K_{0}(x)} \Gamma^{(0)}\left[K_{0} \phi \phi\right]\right\rangle\left\langle\Gamma^{(0)}[J \phi \phi \phi] \frac{\delta \Gamma^{(0)}\left[K_{0} \phi \phi\right]}{\delta \phi_{a}(x)}\right\rangle\right\rangle
\end{gathered}
$$

where all contributions including tadpoles have been discarded.

The second term in the L.H.S. of eq.(133) becomes

$$
\begin{aligned}
& \left\langle g \epsilon_{a b c} \frac{\delta \Gamma^{(0)}[\phi \phi]}{\delta \phi_{b}(x)} \phi_{c}(x) \Gamma^{(0)}[J \phi \phi] \Gamma^{(0)}\left[K_{0} \phi \phi \phi \phi\right] \Gamma^{(0)}\left[K_{0} \phi \phi\right]\right\rangle \\
& =-\left\langle g \epsilon_{a b c} \square \phi_{b}(x) \phi_{c}(x) \Gamma^{(0)}[J \phi \phi] \Gamma^{(0)}\left[K_{0} \phi \phi \phi \phi\right] \Gamma^{(0)}\left[K_{0} \phi \phi\right]\right\rangle \\
& =i\left\langle g \epsilon_{a b c} \frac{\delta \Gamma^{(0)}[J \phi \phi]}{\delta \phi_{b}(x)} \phi_{c}(x) \Gamma^{(0)}\left[K_{0} \phi \phi \phi \phi\right] \Gamma^{(0)}\left[K_{0} \phi \phi\right]\right\rangle
\end{aligned}
$$

since by the functional equation

$$
\begin{aligned}
& g \epsilon_{a b c} \frac{\delta \Gamma^{(0)}\left[K_{0} \phi \phi \phi \phi\right]}{\delta \phi_{b}(x)} \phi_{c}(x)=0, \\
& g \epsilon_{a b c} \frac{\delta \Gamma^{(0)}\left[K_{0} \phi \phi\right]}{\delta \phi_{b}(x)} \phi_{c}(x)=0 .
\end{aligned}
$$


The second term in the L.H.S. of eq.(134) becomes

$$
\begin{aligned}
& \frac{i}{2}\left\langle g \epsilon_{a b c} \frac{\delta \Gamma^{(0)}[\phi \phi]}{\delta \phi_{b}(x)} \phi_{c}(x) \Gamma^{(0)}[J \phi \phi] \Gamma^{(0)}\left[K_{0} \phi \phi\right] \Gamma^{(0)}\left[K_{0} \phi \phi\right] \Gamma^{(0)}[\phi \phi \phi \phi]\right\rangle \\
& =-\frac{i}{2}\left\langle g \epsilon_{a b c} \square \phi_{b}(x) \phi_{c}(x) \Gamma^{(0)}[J \phi \phi] \Gamma^{(0)}\left[K_{0} \phi \phi\right] \Gamma^{(0)}\left[K_{0} \phi \phi\right] \Gamma^{(0)}[\phi \phi \phi \phi]\right\rangle \\
& \quad=-\frac{1}{2}\left\langle g \epsilon_{a b c} \frac{\delta \Gamma^{(0)}[J \phi \phi]}{\delta \phi_{b}(x)} \phi_{c}(x) \Gamma^{(0)}\left[K_{0} \phi \phi\right] \Gamma^{(0)}\left[K_{0} \phi \phi\right] \Gamma^{(0)}[\phi \phi \phi \phi]\right\rangle
\end{aligned}
$$

by the second of eqs.(41) and the equation

$$
g \epsilon_{a b c} \frac{\delta \Gamma^{(0)}[\phi \phi \phi \phi]}{\delta \phi_{b}(x)} \phi_{c}(x)=0
$$

which also follows from the functional equation for $\Gamma^{(0)}$.

It is also convenient to replace the insertion of the composite operator $g \epsilon_{a b c} \frac{\delta \Gamma^{(0)}[J \phi \phi]}{\delta \phi_{b}(x)} \phi_{c}(x)$ in eqs. (40) and (42) with that of

$$
-m_{D} \frac{\delta \Gamma^{(0)}[J \phi \phi \phi]}{\delta \phi_{a}(x)}-\frac{\delta \Gamma^{(0)}\left[K_{0} \phi \phi\right]}{\delta K_{0}(x)} \frac{\delta \Gamma^{(0)}[J \phi]}{\delta \phi_{a}(x)}+2 g \epsilon_{a b c} \frac{\delta \Gamma^{(0)}[J \phi \phi]}{\delta J_{c}^{\mu}(x)} J_{b}^{\mu}(x)
$$

by using eq.(31). We now notice that the relevant unsubtracted amplitudes give rise to the following contractions:

$$
\begin{aligned}
& i \frac{m_{D}^{2}}{2} \partial^{\mu} \frac{\delta}{\delta J_{a}^{\mu}(x)} \Gamma^{(2,0)}[J\left.J K_{0} K_{0}\right]=\frac{1}{2}\left\langle\frac{m_{D}^{2}}{2} \partial^{\mu} \frac{\delta \Gamma^{(0)}[J \phi \phi \phi]}{\delta J_{a}^{\mu}(x)} \Gamma^{(0)}[J \phi \phi \phi] \Gamma^{(0)}\left[K_{0} \phi \phi\right] \Gamma^{(0)}\left[K_{0} \phi \phi\right]\right\rangle \\
&+\left\langle\frac{m_{D}^{2}}{2} \partial^{\mu} \frac{\delta \Gamma^{(0)}[J \phi \phi]}{\delta J_{a}^{\mu}(x)} \Gamma^{(0)}[J \phi \phi] \Gamma^{(0)}\left[K_{0} \phi \phi \phi \phi\right] \Gamma^{(0)}\left[K_{0} \phi \phi\right]\right\rangle \\
&+ \frac{i}{2}\left\langle\frac{m_{D}^{2}}{2} \partial^{\mu} \frac{\delta \Gamma^{(0)}[J \phi \phi]}{\delta J_{a}^{\mu}(x)} \Gamma^{(0)}[J \phi \phi] \Gamma^{(0)}\left[K_{0} \phi \phi\right] \Gamma^{(0)}\left[K_{0} \phi \phi\right] \Gamma^{(0)}[\phi \phi \phi \phi]\right\rangle \\
&-\left\langle\left\langle\frac{m_{D}^{2}}{2} \partial^{\mu} \frac{\delta \Gamma^{(0)}[J \phi \phi \phi]}{\delta J_{a}^{\mu}(x)} \Gamma^{(0)}\left[K_{0} \phi \phi\right]\right\rangle\left\langle\Gamma^{(0)}[J \phi \phi \phi] \Gamma^{(0)}\left[K_{0} \phi \phi\right]\right\rangle\right\rangle, \\
& i m_{D} \frac{\delta}{\delta \phi_{a}(x)} \Gamma^{(2,0)}\left[\phi J K_{0} K_{0}\right]= \frac{1}{2}\left\langle m_{D} \frac{\delta \Gamma^{(0)}[\phi \phi \phi \phi]}{\delta \phi_{a}(x)} \Gamma^{(0)}\left[K_{0} \phi \phi\right] \Gamma^{(0)}\left[K_{0} \phi \phi\right] \Gamma^{(0)}[J \phi \phi \phi]\right\rangle \\
&+\frac{1}{2}\left\langle\Gamma^{(0)}[\phi \phi \phi \phi] \Gamma^{(0)}\left[K_{0} \phi \phi\right] \Gamma^{(0)}\left[K_{0} \phi \phi\right] m_{D} \frac{\delta \Gamma^{(0)}[J \phi \phi \phi]}{\delta \phi_{a}(x)}\right\rangle \\
&-i\left\langle m_{D} \frac{\delta \Gamma^{(0)}\left[K_{0} \phi \phi \phi \phi\right]}{\delta \phi_{a}(x)} \Gamma^{(0)}\left[K_{0} \phi \phi\right] \Gamma^{(0)}[J \phi \phi \phi]\right\rangle \\
&-i\left\langle\Gamma^{(0)}\left[K_{0} \phi \phi \phi \phi\right] \Gamma^{(0)}\left[K_{0} \phi \phi\right] m_{D} \frac{\delta \Gamma^{(0)}[J \phi \phi \phi]}{\delta \phi_{a}(x)}\right\rangle
\end{aligned}
$$




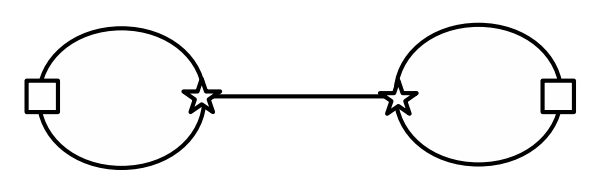

Figure 2: Spurious (non 1-PI) contribution to $\Gamma^{(2,0)}\left[J J K_{0} K_{0}\right]$.

$$
\begin{aligned}
& -\frac{i}{2}\left\langle m_{D} \frac{\delta \Gamma^{(0)}[J \phi \phi \phi \phi \phi]}{\delta \phi_{a}(x)} \Gamma^{(0)}\left[K_{0} \phi \phi\right] \Gamma^{(0)}\left[K_{0} \phi \phi\right]\right\rangle \\
& -\left\langle\left\langle m_{D} \frac{\delta \Gamma^{(0)}[J \phi \phi \phi]}{\delta \phi_{a}(x)} \Gamma^{(0)}\left[K_{0} \phi \phi\right]\right\rangle\left\langle\Gamma^{(0)}[\phi \phi \phi \phi] \Gamma^{(0)}\left[K_{0} \phi \phi\right]\right\rangle\right\rangle \\
& -\left\langle\left\langle\Gamma^{(0)}[J \phi \phi \phi] \Gamma^{(0)}\left[K_{0} \phi \phi\right]\right\rangle\left\langle m_{D} \frac{\delta \Gamma^{(0)}[\phi \phi \phi \phi]}{\delta \phi_{a}(x)} \Gamma^{(0)}\left[K_{0} \phi \phi\right]\right\rangle\right\rangle
\end{aligned}
$$

and

$$
\begin{aligned}
& i \frac{\delta \Gamma^{(1,0)}\left[K_{0} K_{0}\right]}{\delta K_{0}(x)} \frac{\delta \Gamma^{(1,0)}\left[\phi K_{0} J\right]}{\delta \phi_{a}(x)}= \\
&-i\left\langle\frac{\delta \Gamma^{(0)}\left[K_{0} \phi \phi\right]}{\delta K_{0}(x)} \Gamma^{(0)}\left[K_{0} \phi \phi\right]\right\rangle\left\langle\frac{\delta \Gamma^{(0)}[J \phi \phi \phi]}{\delta \phi_{a}(x)} \Gamma^{(0)}\left[K_{0} \phi \phi\right]\right\rangle, \\
& i \frac{\delta \Gamma^{(2,0)}\left[K_{0} K_{0} K_{0}\right]}{\delta K_{0}(x)} \frac{\delta \Gamma^{(0)}[\phi J]}{\delta \phi_{a}(x)}= \\
& \frac{1}{2}\left\langle\Gamma^{(0)}[\phi \phi \phi \phi] \frac{\delta \Gamma^{(0)}\left[K_{0} \phi \phi\right]}{\delta K_{0}(x)} \Gamma^{(0)}\left[K_{0} \phi \phi\right] \Gamma^{(0)}\left[K_{0} \phi \phi\right]\right\rangle \frac{\delta \Gamma^{(0)}[\phi J]}{\delta \phi_{a}(x)} \\
& \quad-\frac{i}{2}\left\langle\frac{\delta \Gamma^{(0)}\left[K_{0} \phi \phi \phi \phi\right]}{\delta K_{0}(x)} \Gamma^{(0)}\left[K_{0} \phi \phi\right] \Gamma^{(0)}\left[K_{0} \phi \phi\right]\right\rangle \frac{\delta \Gamma^{(0)}[\phi J]}{\delta \phi_{a}(x)} \\
&-i\left\langle\Gamma^{(0)}\left[K_{0} \phi \phi \phi \phi\right] \frac{\delta \Gamma^{(0)}\left[K_{0} \phi \phi\right]}{\delta K_{0}(x)} \Gamma^{(0)}\left[K_{0} \phi \phi\right]\right\rangle \frac{\delta \Gamma^{(0)}[\phi J]}{\delta \phi_{a}(x)} .
\end{aligned}
$$

The last terms in eq.(44) and in eq. (45) subtract the connected but not 1-PI contributions, shown in Figure 2 and 3 that enter in the first term on the R.H.S. of eqs. (44) and (45) respectively.

By using eqs.(38), (39), (40) and (42) and the fact that $\Gamma^{(2,0)}\left[J K_{0} K_{0}\right]$ is zero by $S U(2)$ global symmetry a straightforward computation shows that 


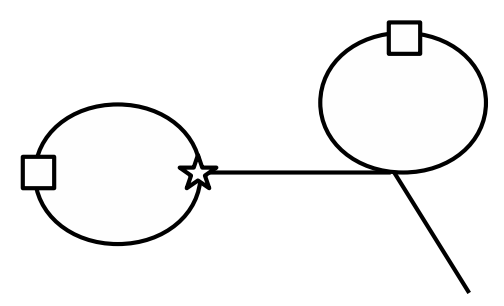

Figure 3: Spurious (non 1-PI) contribution to $\Gamma^{(2,0)}\left[\phi J K_{0} K_{0}\right]$.

the sum of eqs. (32)-

$$
\begin{aligned}
& i \frac{m_{D}^{2}}{2} \partial^{\mu} \frac{\delta \Gamma^{(2,0)}\left[J J K_{0} K_{0}\right]}{\delta J_{a}^{\mu}(x)}+i m_{D} \frac{\delta \Gamma^{(2,0)}\left[\phi J K_{0} K_{0}\right]}{\delta \phi_{a}(x)} \\
& +i \frac{\delta \Gamma^{(1,0)}\left[K_{0} K_{0}\right]}{\delta K_{0}(x)} \frac{\delta \Gamma^{(1,0)}\left[\phi K_{0} J\right]}{\delta \phi_{a}(x)}+i \frac{\delta \Gamma^{(2,0)}\left[K_{0} K_{0} K_{0}\right]}{\delta K_{0}(x)} \frac{\delta \Gamma^{(0)}[\phi J]}{\delta \phi_{a}(x)}=0,
\end{aligned}
$$

i.e. the functional equation for the unsubtracted amplitudes.

\section{B Diagrammatic analysis of the functional equa- tion for amplitudes with one counterterm inser- tion}

We evaluate the insertions in eq.(21) and eq.(23) in the relevant sector spanned by two $K_{0}$ and one $J_{\mu}$.

The one-loop functional equation for the counterterms (18) yields

$$
\begin{aligned}
& m_{D} \frac{\delta \hat{\Gamma}^{(1)}\left[K_{0} J \phi \phi \phi\right]}{\delta \phi_{a}(x)}+\frac{\delta \hat{\Gamma}^{(1)}\left[K_{0} K_{0}\right]}{\delta K_{0}(x)} \frac{\delta \Gamma^{(0)}[\phi \phi \phi J]}{\delta \phi_{a}(x)}+\frac{\delta \hat{\Gamma}^{(1)}\left[\phi K_{0} J\right]}{\delta K_{0}(x)} \frac{\delta \Gamma^{(0)}\left[\phi \phi K_{0}\right]}{\delta \phi_{a}(x)} \\
& +\frac{\delta \Gamma^{(0)}\left[\phi \phi K_{0}\right]}{\delta K_{0}(x)} \frac{\delta \hat{\Gamma}^{(1)}\left[\phi K_{0} J\right]}{\delta \phi_{a}(x)}+\frac{\delta \hat{\Gamma}^{(1)}\left[K_{0} K_{0} \phi \phi\right]}{\delta K_{0}(x)} \frac{\delta \Gamma^{(0)}\left[\phi_{a} J\right]}{\delta \phi_{a}(x)}+\frac{m_{D}^{2}}{2} \partial^{\mu} \frac{\delta \hat{\Gamma}^{(1)}\left[K_{0} J J \phi \phi\right]}{\delta J_{a}^{\mu}(x)} \\
& -2 g \epsilon_{a b c} \frac{\delta \hat{\Gamma}^{(1)}\left[K_{0} J \phi \phi\right]}{\delta J_{c}^{\mu}(x)} J_{b}^{\mu}(x)+g \epsilon_{a b c} \frac{\delta \hat{\Gamma}^{(1)}\left[K_{0} J \phi \phi\right]}{\delta \phi_{b}(x)} \phi_{c}(x)=0 \\
& m_{D} \frac{\delta \hat{\Gamma}^{(1)}[J \phi \phi \phi]}{\delta \phi_{a}(x)}+\frac{\delta \hat{\Gamma}^{(1)}\left[K_{0} J \phi\right]}{\delta K_{0}(x)} \frac{\delta \Gamma^{(0)}[\phi \phi]}{\delta \phi_{a}(x)}+\frac{\delta \hat{\Gamma}^{(1)}\left[K_{0} \phi \phi\right]}{\delta K_{0}(x)} \frac{\delta \Gamma^{(0)}[\phi J]}{\delta \phi_{a}(x)} \\
& +\frac{m_{D}^{2}}{2} \partial^{\mu} \frac{\delta \hat{\Gamma}^{(1)}[J J \phi \phi]}{\delta J_{a}^{\mu}(x)}-2 g \epsilon_{a b c} \frac{\delta \hat{\Gamma}^{(1)}[J \phi \phi]}{\delta J_{c}^{\mu}(x)} J_{b}^{\mu}(x)+g \epsilon_{a b c} \frac{\delta \hat{\Gamma}^{(1)}[J \phi \phi]}{\delta \phi_{b}(x)} \phi_{c}(x)=0
\end{aligned}
$$


The zero-loop functional equation (11) yields

$$
\begin{aligned}
& m_{D} \frac{\delta \Gamma^{(0)}[J \phi \phi \phi]}{\delta \phi_{a}(x)}-2 g \epsilon_{a b c} \frac{\delta \Gamma^{(0)}[J \phi \phi]}{\delta J_{c}^{\mu}(x)} J_{b}^{\mu}(x) \\
& +g \epsilon_{a b c} \frac{\delta \Gamma^{(0)}[J \phi \phi]}{\delta \phi_{b}(x)} \phi_{c}(x)+\frac{\delta \Gamma^{(0)}\left[K_{0} \phi \phi\right]}{\delta K_{0}(x)} \frac{\delta \Gamma^{(0)}[J \phi]}{\delta \phi_{a}(x)}=0 .
\end{aligned}
$$

The insertion of eq. (49) gives

$$
\begin{aligned}
& -\left\langle\left( m_{D} \frac{\delta \hat{\Gamma}^{(1)}\left[K_{0} J \phi \phi \phi\right]}{\delta \phi_{a}(x)}+\frac{\delta \hat{\Gamma}^{(1)}\left[K_{0} K_{0}\right]}{\delta K_{0}(x)} \frac{\delta \Gamma^{(0)}[\phi \phi \phi J]}{\delta \phi_{a}(x)}+\frac{\delta \hat{\Gamma}^{(1)}\left[\phi K_{0} J\right]}{\delta K_{0}(x)} \frac{\delta \Gamma^{(0)}\left[\phi \phi K_{0}\right]}{\delta \phi_{a}(x)}\right.\right. \\
& +\frac{\delta \Gamma^{(0)}\left[\phi \phi K_{0}\right]}{\delta K_{0}(x)} \frac{\delta \hat{\Gamma}^{(1)}\left[\phi K_{0} J\right]}{\delta \phi_{a}(x)}+\frac{\delta \hat{\Gamma}^{(1)}\left[K_{0} K_{0} \phi \phi\right]}{\delta K_{0}(x)} \frac{\delta \Gamma^{(0)}\left[\phi_{a} J\right]}{\delta \phi_{a}(x)}+\frac{m_{D}^{2}}{2} \partial^{\mu} \frac{\delta \hat{\Gamma}^{(1)}\left[K_{0} J J \phi \phi\right]}{\delta J_{a}^{\mu}(x)} \\
& \left.\left.-2 g \epsilon_{a b c} \frac{\delta \hat{\Gamma}^{(1)}\left[K_{0} J \phi \phi\right]}{\delta J_{c}^{\mu}(x)} J_{b}^{\mu}(x)+g \epsilon_{a b c} \frac{\delta \hat{\Gamma}^{(1)}\left[K_{0} J \phi \phi\right]}{\delta \phi_{b}(x)} \phi_{c}(x)\right) \Gamma^{(0)}\left[K_{0} \phi \phi\right]\right\rangle=0
\end{aligned}
$$

Moreover the insertion in eq. (150) yields

$$
\begin{aligned}
-\frac{i}{2}\left\langle\left( m_{D} \frac{\delta \hat{\Gamma}^{(1)}[J \phi \phi \phi]}{\delta \phi_{a}(x)}+\frac{\delta \hat{\Gamma}^{(1)}\left[K_{0} J \phi\right]}{\delta K_{0}(x)} \frac{\delta \Gamma^{(0)}[\phi \phi]}{\delta \phi_{a}(x)}+\frac{\delta \hat{\Gamma}^{(1)}\left[K_{0} \phi \phi\right]}{\delta K_{0}(x)} \frac{\delta \Gamma^{(0)}[\phi J]}{\delta \phi_{a}(x)}\right.\right. \\
+\frac{m_{D}^{2}}{2} \partial^{\mu} \frac{\delta \hat{\Gamma}^{(1)}[J J \phi \phi]}{\delta J_{a}^{\mu}(x)}-2 g \epsilon_{a b c} \frac{\delta \hat{\Gamma}^{(1)}[J \phi \phi]}{\delta J_{c}^{\mu}(x)} J_{b}^{\mu}(x) \\
\left.\left.+g \epsilon_{a b c} \frac{\delta \hat{\Gamma}^{(1)}[J \phi \phi]}{\delta \phi_{b}(x)} \phi_{c}(x)\right) \Gamma^{(0)}\left[K_{0} \phi \phi\right] \Gamma^{(0)}\left[K_{0} \phi \phi\right]\right\rangle=0
\end{aligned}
$$

The insertion in eq.(51) finally gives

$$
\begin{aligned}
& -\left\langle\left( m_{D} \frac{\delta \Gamma^{(0)}[J \phi \phi \phi]}{\delta \phi_{a}(x)}-2 g \epsilon_{a b c} \frac{\delta \Gamma^{(0)}[J \phi \phi]}{\delta J_{c}^{\mu}(x)} J_{b}^{\mu}(x)\right.\right. \\
& \left.\left.+g \epsilon_{a b c} \frac{\delta \Gamma^{(0)}[J \phi \phi]}{\delta \phi_{b}(x)} \phi_{c}(x)+\frac{\delta \Gamma^{(0)}\left[K_{0} \phi \phi\right]}{\delta K_{0}(x)} \frac{\delta \Gamma^{(0)}[J \phi]}{\delta \phi_{a}(x)}\right) \hat{\Gamma}^{(1)}\left[K_{0} K_{0} \phi \phi\right]\right\rangle=0
\end{aligned}
$$

and

$$
\begin{aligned}
& -i\left\langle\left( m_{D} \frac{\delta \Gamma^{(0)}[J \phi \phi \phi]}{\delta \phi_{a}(x)}-2 g \epsilon_{a b c} \frac{\delta \Gamma^{(0)}[J \phi \phi]}{\delta J_{c}^{\mu}(x)} J_{b}^{\mu}(x)\right.\right. \\
& \left.\left.+g \epsilon_{a b c} \frac{\delta \Gamma^{(0)}[J \phi \phi]}{\delta \phi_{b}(x)} \phi_{c}(x)+\frac{\delta \Gamma^{(0)}\left[K_{0} \phi \phi\right]}{\delta K_{0}(x)} \frac{\delta \Gamma^{(0)}[J \phi]}{\delta \phi_{a}(x)}\right) \hat{\Gamma}^{(1)}\left[K_{0} \phi \phi\right] \Gamma^{(0)}\left[K_{0} \phi \phi\right]\right\rangle=0 .
\end{aligned}
$$


The second term in the L.H.S. of eq.(152) yields

$$
i \frac{\delta \hat{\Gamma}^{(1)}\left[K_{0} K_{0}\right]}{\delta K_{0}(x)} \frac{\delta \Gamma^{(1,0)}\left[J K_{0} \phi\right]}{\delta \phi_{a}(x)}
$$

since

$$
i \frac{\delta \Gamma^{(1,0)}\left[J K_{0} \phi\right]}{\delta \phi_{a}(x)}=-\left\langle\frac{\delta \Gamma^{(0)}[J \phi \phi \phi]}{\delta \phi_{a}(x)} \Gamma^{(0)}\left[K_{0} \phi \phi\right]\right\rangle .
$$

The fourth term in the L.H.S. eq.(52) yields

$$
i \frac{\delta \hat{\Gamma}^{(1)}\left[J K_{0} \phi\right]}{\delta \phi_{a}(x)} \frac{\delta \Gamma^{(1,0)}\left[K_{0} K_{0}\right]}{\delta K_{0}(x)}
$$

since

$$
i \frac{\delta \Gamma^{(1,0)}\left[K_{0} K_{0}\right]}{\delta K_{0}(x)}=-\left\langle\frac{\delta \Gamma^{(0)}\left[K_{0} \phi \phi\right]}{\delta K_{0}(x)} \Gamma^{(0)}\left[K_{0} \phi \phi\right]\right\rangle .
$$

The terms proportional to $\frac{\delta \Gamma^{(0)}[J \phi]}{\delta \phi_{a}(x)}$ in eqs.(152), (153), (154) and (155) yield

$$
i \frac{\delta \Gamma^{(2,1)}\left[K_{0} K_{0} K_{0}\right]}{\delta K_{0}(x)} \frac{\delta \Gamma^{(0)}[\phi J]}{\delta \phi_{a}(x)}
$$

as one can see by taking the derivative w.r.t. $K_{0}(x)$ of

$$
\begin{aligned}
i \Gamma^{(2,1)}\left[K_{0} K_{0} K_{0}\right]= & -\left\langle\hat{\Gamma}^{(1)}\left[K_{0} K_{0} \phi \phi\right] \Gamma^{(0)}\left[K_{0} \phi \phi\right]\right\rangle \\
& -\frac{i}{2}\left\langle\hat{\Gamma}^{(1)}\left[K_{0} \phi \phi\right] \Gamma^{(0)}\left[K_{0} \phi \phi\right] \Gamma^{(0)}\left[K_{0} \phi \phi\right]\right\rangle .
\end{aligned}
$$

The second term in the L.H.S. of eq. (53) contains $\frac{\delta \Gamma^{(0)}[\phi \phi]}{\delta \phi_{a}(x)}=-\square \phi_{a}(x)$. By making use of eq.(37) it can be easily checked by a direct computation that the amplitudes involving the second term in the L.H.S. of eq. (53) cancel out with those involving the third term in eq.(52).

We now evaluate the two-loop amplitudes corrected with the one-loop counterterms which enter in the first line of eq.(15):

$$
\begin{aligned}
i \frac{m_{D}^{2}}{2} \partial^{\mu} \frac{\delta \Gamma^{(2,1)}\left[J J K_{0} K_{0}\right]}{\delta J_{a}^{\mu}}= & -\left\langle\frac{m_{D}^{2}}{2} \partial^{\mu} \frac{\delta \hat{\Gamma}^{(1)}\left[J J K_{0} \phi \phi\right]}{\delta J_{a}^{\mu}} \Gamma^{(0)}\left[K_{0} \phi \phi\right]\right\rangle \\
& -\frac{i}{2}\left\langle\frac{m_{D}^{2}}{2} \partial^{\mu} \frac{\delta \hat{\Gamma}^{(1)}[J J \phi \phi]}{\delta J_{a}^{\mu}} \Gamma^{(0)}\left[K_{0} \phi \phi\right] \Gamma^{(0)}\left[K_{0} \phi \phi\right]\right\rangle \\
& -i\left\langle\frac{m_{D}^{2}}{2} \partial^{\mu} \frac{\delta \hat{\Gamma}^{(1)}\left[J K_{0} \phi \phi\right]}{\delta J_{a}^{\mu}} \Gamma^{(0)}[J \phi \phi] \Gamma^{(0)}\left[K_{0} \phi \phi\right]\right\rangle
\end{aligned}
$$




$$
\begin{aligned}
& -i\left\langle\hat{\Gamma}^{(1)}\left[J K_{0} \phi \phi\right] \frac{m_{D}^{2}}{2} \partial^{\mu} \frac{\delta \Gamma^{(0)}[J \phi \phi]}{\delta J_{a}^{\mu}} \Gamma^{(0)}\left[K_{0} \phi \phi\right]\right\rangle \\
& -i\left\langle\hat{\Gamma}^{(1)}\left[K_{0} K_{0} \phi \phi\right] \frac{m_{D}^{2}}{2} \partial^{\mu} \frac{\delta \Gamma^{(0)}[J \phi \phi]}{\delta J_{a}^{\mu}} \Gamma^{(0)}[J \phi \phi]\right\rangle \\
& +\frac{1}{2}\left\langle\frac{m_{D}^{2}}{2} \partial^{\mu} \frac{\delta \hat{\Gamma}^{(1)}[J \phi \phi]}{\delta J_{a}^{\mu}} \Gamma^{(0)}[J \phi \phi] \Gamma^{(0)}\left[K_{0} \phi \phi\right] \Gamma^{(0)}\left[K_{0} \phi \phi\right]\right\rangle \\
& +\frac{1}{2}\left\langle\hat{\Gamma}^{(1)}[J \phi \phi] \frac{m_{D}^{2}}{2} \partial^{\mu} \frac{\delta \Gamma^{(0)}[J \phi \phi]}{\delta J_{a}^{\mu}} \Gamma^{(0)}\left[K_{0} \phi \phi\right] \Gamma^{(0)}\left[K_{0} \phi \phi\right]\right\rangle \\
& +\left\langle\hat{\Gamma}^{(1)}\left[K_{0} \phi \phi\right] \frac{m_{D}^{2}}{2} \partial^{\mu} \frac{\delta \Gamma^{(0)}[J \phi \phi]}{\delta J_{a}^{\mu}} \Gamma^{(0)}[J \phi \phi] \Gamma^{(0)}\left[K_{0} \phi \phi\right]\right\rangle, \\
i m_{D} \frac{\delta \Gamma^{(2,1)}\left[\phi J K_{0} K_{0}\right]}{\delta \phi_{a}(x)}=\quad & -\left\langle\Gamma^{(0)}\left[K_{0} \phi \phi\right] m_{D} \frac{\delta \hat{\Gamma}^{(1)}\left[K_{0} J \phi \phi \phi\right]}{\delta \phi_{a}(x)}\right\rangle \\
- & \frac{i}{2}\left\langle\Gamma^{(0)}\left[K_{0} \phi \phi\right] \Gamma^{(0)}\left[K_{0} \phi \phi\right] m_{D} \frac{\delta \hat{\Gamma}^{(1)}[J \phi \phi \phi]}{\delta \phi_{a}(x)}\right\rangle \\
- & \left\langle m_{D} \frac{\delta \Gamma^{(0)}[J \phi \phi \phi]}{\delta \phi_{a}(x)} \hat{\Gamma}^{(1)}\left[K_{0} K_{0} \phi \phi\right]\right\rangle \\
- & i\left\langle m_{D} \frac{\delta \Gamma^{(0)}[J \phi \phi \phi]}{\delta \phi_{a}(x)} \Gamma^{(0)}\left[K_{0} \phi \phi\right] \hat{\Gamma}^{(1)}\left[K_{0} \phi \phi\right]\right\rangle .
\end{aligned}
$$

In order to proceed further one needs to use eq.(28) into eq.(62). This allows to replace the insertion of

$$
\frac{m_{D}^{2}}{2} \partial^{\mu} \frac{\delta \Gamma^{(0)}[J \phi \phi]}{\delta J_{a}^{\mu}}
$$

with that of

$$
-g \epsilon_{a b c} \frac{\delta \Gamma^{(0)}[\phi \phi]}{\delta \phi_{b}(x)} \phi_{c}(x)=g \epsilon_{a b c} \square \phi_{b}(x) \phi_{c}(x) .
$$

One can then perform in a straightforward way the relevant contractions with the help of eq. (37). It is also convenient to use the fact that, as a consquence of the one-loop functional equation, the following identities hold:

$$
\frac{m_{D}^{2}}{2} \partial_{\mu} \frac{\delta \hat{\Gamma}^{(1)}\left[K_{0} J \phi \phi\right]}{\delta J_{a}^{\mu}(x)}+g \epsilon_{a b c} \frac{\delta \hat{\Gamma}^{(1)}\left[K_{0} \phi \phi\right]}{\delta \phi_{b}(x)} \phi_{c}(x)=0
$$

and

$$
\frac{m_{D}^{2}}{2} \partial_{\mu} \frac{\delta \hat{\Gamma}^{(1)}[J \phi \phi]}{\delta J_{a}^{\mu}(x)}=0
$$


In the above equation we have used the fact that $\hat{\Gamma}_{\phi^{a} \phi^{b}}^{(1)}=0$ (see Refs. [1], [2]).

Therefore one obtains

$$
\begin{aligned}
i \frac{m_{D}^{2}}{2} \partial^{\mu} \frac{\delta \Gamma^{(2,1)}\left[J J K_{0} K_{0}\right]}{\delta J_{a}^{\mu}(x)}= & -\left\langle\frac{m_{D}^{2}}{2} \partial^{\mu} \frac{\delta \hat{\Gamma}^{(1)}\left[J J K_{0} \phi \phi\right]}{\delta J_{a}^{\mu}(x)} \Gamma^{(0)}\left[K_{0} \phi \phi\right]\right\rangle \\
& -\frac{i}{2}\left\langle\frac{m_{D}^{2}}{2} \partial^{\mu} \frac{\delta \hat{\Gamma}^{(1)}[J J \phi \phi]}{\delta J_{a}^{\mu}(x)} \Gamma^{(0)}\left[K_{0} \phi \phi\right] \Gamma^{(0)}\left[K_{0} \phi \phi\right]\right\rangle \\
& -\left\langle g \epsilon_{a b c} \frac{\delta \hat{\Gamma}^{(1)}\left[J K_{0} \phi \phi\right]}{\delta \phi_{b}(x)} \phi_{c}(x) \Gamma^{(0)}\left[K_{0} \phi \phi\right]\right\rangle \\
& -\left\langle g \epsilon_{a b c} \frac{\delta \Gamma^{(0)}[J \phi \phi]}{\delta \phi_{b}(x)} \phi_{c}(x) \hat{\Gamma}^{(1)}\left[K_{0} K_{0} \phi \phi\right]\right\rangle \\
& -\frac{i}{2}\left\langle g \epsilon_{a b c} \frac{\delta \hat{\Gamma}^{(1)}[J \phi \phi]}{\delta \phi_{b}(x)} \phi_{c}(x) \Gamma^{(0)}\left[K_{0} \phi \phi\right] \Gamma^{(0)}\left[K_{0} \phi \phi\right]\right\rangle \\
& -i\left\langle g \epsilon_{a b c} \frac{\delta \Gamma^{(0)}[J \phi \phi]}{\delta \phi_{b}(x)} \phi_{c}(x) \hat{\Gamma}^{(1)}\left[K_{0} \phi \phi\right] \Gamma^{(0)}\left[K_{0} \phi \phi\right]\right\rangle .
\end{aligned}
$$

By taking into account eqs.(56), (58), (60), (63) and (66) and the fact that $\Gamma^{(2,1)}\left[K_{0} K_{0} J\right]$ is zero by $S U(2)$ global symmetry the sum of eqs.(152),(153),(154) and (55) yields finally

$$
\begin{aligned}
& i\left(\frac{m_{D}^{2}}{2} \partial^{\mu} \Gamma_{J_{a}^{\mu}}^{(2,1)}\left[J J K_{0} K_{0}\right]+m_{D} \Gamma_{\phi_{a}}^{(2,1)}\left[K_{0} K_{0} J \phi\right]\right. \\
& +\Gamma_{K_{0}}^{(2,1)}\left[K_{0} K_{0} K_{0}\right] \Gamma_{\phi_{a}}^{(0)}[\phi J]+\Gamma_{K_{0}}^{(1,0)}\left[K_{0} K_{0}\right] \hat{\Gamma}_{\phi_{a}}^{(1)}\left[\phi K_{0} J\right] \\
& \left.+\hat{\Gamma}_{K_{0}}^{(1)}\left[K_{0} K_{0}\right] \Gamma_{\phi_{a}}^{(1,0)}\left[J K_{0} \phi\right]\right)=0 .
\end{aligned}
$$

\section{References}

[1] R. Ferrari, JHEP 0508 (2005) 048 arXiv:hep-th/0504023.

[2] R. Ferrari and A. Quadri, arXiv:hep-th/0506220.

[3] G. Ecker and J. Honerkamp, Nucl. Phys. B 35 (1971) 481.

[4] T. Appelquist and C. W. Bernard, Phys. Rev. D 23 (1981) 425.

[5] L. D. Faddeev and A. A. Slavnov, Lett. Nuovo Cimento 8 (1973) 117.

[6] L. Tataru, Phys. Rev. D 12 (1975) 3351.

[7] I. S. Gerstein, R. Jackiw, S. Weinberg and B. W. Lee, Phys. Rev. D 3 (1971) 2486. 
[8] J. M. Charap, Phys. Rev. D 2 (1970) 1554.

[9] J. Honerkamp and K. Meetz, Phys. Rev. D 3 (1971) 1996.

[10] P. Breitenlohner and D. Maison, Commun. Math. Phys. 52 (1977) 11.

[11] P. Breitenlohner and D. Maison, Commun. Math. Phys. 52 (1977) 39.

[12] P. Breitenlohner and D. Maison, Commun. Math. Phys. 52 (1977) 55.

[13] E. Brezin, J. Zinn-Justin and J. C. Le Guillou, Phys. Rev. D 14 (1976) 2615.

[14] D. H. Friedan, Annals Phys. 163 (1985) 318.

[15] L. Alvarez-Gaume, D. Z. Freedman and S. Mukhi, Annals Phys. 134 (1981) 85 . 